ACCepted for publichtion in the Astrophysical Journal

Preprint typeset using $\mathrm{LATEX}_{\mathrm{E}}$ style emulateapj v. 08/13/06

\title{
OPTICAL SPECTROPOLARIMETRY AND ASPHERICITY OF TYPE Ic SN 2007gr ${ }^{1}$
}

\author{
Masaomi Tanaka ${ }^{2}$, Koji S. Kawabata ${ }^{3}$, Keilchi Maeda ${ }^{4}$, Takashi Hattori ${ }^{5}$, and Ken'ichi Nomoto ${ }^{4,2}$
}

\begin{abstract}
We present optical spectropolarimetric observations of Type Ic supernova (SN) 2007gr with Subaru telescope at 21 days after the maximum brightness ( $\sim 37$ days after the explosion). Non-zero polarization as high as $\sim 3 \%$ is observed at the absorption feature of Ca II IR triplet. The polarization of the continuum light is $\sim 0.5 \%$ if we estimate the interstellar polarization (ISP) component assuming that the continuum polarization has a single polarization angle. This suggests that the axis ratio of the SN photosphere projected to the sky is different from unity by $\sim 10 \%$. The polarization angle at the Ca II absorption is almost aligned to that of the continuum light. These features may be understood by the model where a bipolar explosion with an oblate photosphere is viewed from the slightly off-axis direction and explosively synthesized $\mathrm{Ca}$ near the polar region obscures the light originated around the minor axis of the SN photosphere. Given the uncertainty of the ISP, however, the polarization data could also be interpreted by the model with an almost spherically symmetric photosphere and a clumpy Ca II distribution.

Subject headings: polarization — supernovae: general — supernovae: individual (SN 2007gr)
\end{abstract}

\section{INTRODUCTION}

The explosion mechanism of core-collapse supernovae (SNe) is still under debate. Recent theoretical studies show that the effects causing non-spherical explosion, such as magnetic field, rotation, and several kinds of instabilities, are important for the successful explosion (e.g., Blondin et al. 2003; Kotake et al. 2004; Buras et al. 2006; Burrows et al. 2006).

In these circumstances, observational constraints on the SN asymmetry are important. Such observations include the direct imaging of Galactic young supernova remnants (e.g., Hwang et al. 2004) and SN 1987A (Wang et al. 2002), although the number of such objects is limited. The observations of extragalactic, point-source $\mathrm{SNe}$ also give clues to the explosion geometry. For example, the shape of emission lines in optical spectra at $\gtrsim 1$ year after the explosion can be used to study the multi-dimensional structure in the innermost part of core-collapse SNe (Mazzali et al. 2005; Maeda et al. 2008; Modjaz et al. 2008).

The most direct way to study the asymmetry of extragalactic, point-source SNe at early phases is polarimetry. Since the polarized lights scattered by electrons in the ejecta are completely cancelled out in the spherically symmetric case, the detection of polarization undoubtedly indicates the deviation from spherical symmetry (Shapiro \& Sutherland 1982; Höflich 1991). With spectropolarimetry, the polarization across the P-Cygni profile can give the information on the distribution of elements. Spectropolarimetric studies have clarified the

\footnotetext{
${ }^{1}$ Based on data collected at Subaru Telescope, which is operated by the National Astronomical Observatory of Japan.

${ }^{2}$ Department of Astronomy, Graduate School of Science, University of Tokyo, 7-3-1 Hongo, Bunkyo-ku, Tokyo 113-0033, Japan; mtanaka@astron.s.u-tokyo.ac.jp

${ }^{3}$ Hiroshima Astrophysical Science Center, Hiroshima University, Higashi-Hiroshima, Hiroshima, Japan

${ }^{4}$ Institute for the Physics and Mathematics of the Universe, University of Tokyo, Kashiwa, Japan

${ }^{5}$ Subaru Telescope, National Astronomical Observatory of Japan, Hilo, HI
}

asymmetric nature of core-collapse $\mathrm{SNe}$ in detail (e.g., Cropper et al. 1988; Trammell, Hines \& Wheeler 1993; Wang et al. 2001; Leonard et al. 2001, 2006; Kawabata et al. 2002).

In this paper, we present the spectropolarimetric observation of SN 2007gr. SN 2007gr was discovered in NGC 1058 (Li et al. 2007). Thanks to the short distance to this host galaxy ${ }^{6}$, SN 2007 gr reached 12.7 mag in the $\mathrm{R}$ band at maximum brightness (Valenti et al. 2008), making the spectropolarimetric observation possible. Photometric and spectroscopic properties of SN 2007gr were shown by Valenti et al. (2008). They classified this SN as Type Ic due to the absence of the $\mathrm{H}$ and He lines. The progenitor of Type Ic SNe is thought to have lost its $\mathrm{H}$ and He layers before the explosion (e.g., Wheeler et al. 1987; Nomoto et al. 1994). Non-detection in the pre-explosion Hubble Space Telescope image at the SN position is consistent with a Wolf-Rayet star progenitor (Crockett et al. 2008). The maximum brightness of SN 2007gr is similar to that of normal SNe, being powered by the decay of $\sim 0.07-0.1 M_{\odot}{ }^{56} \mathrm{Ni}$ (Valenti et al. 2008).

We present in $\S 2$ the observation and data reduction of SN 2007gr. Results of our spectropolarimetric observation are shown in $\S 3$, where the interstellar polarization (ISP) is also discussed. We study the multi-dimensional explosion geometry of SN 2007gr in $\S 4$ and summarize conclusions in $\S 5$.

\section{OBSERVATIONS AND DATA REDUCTION}

The spectropolarimetric observation of SN 2007gr was performed with the $8.2 \mathrm{~m}$ Subaru telescope equipped with the Faint Object Camera and Spectrograph (FOCAS, Kashikawa et al. 2002) on 2007 September 18 UT. This epoch corresponds to 21 days after the maximum brightness, which is $\sim 37$ days after the explosion assum-

${ }^{6}$ Crockett et al. (2008) adopted 10.6 Mpc for the distance to the SN (Schmidt et al. 1992; Terry et al. 2002; Pilyugin et al. 2004) while Valenti et al. (2008) adopted 9.3 Mpc (Silbermann et al. 1996). 


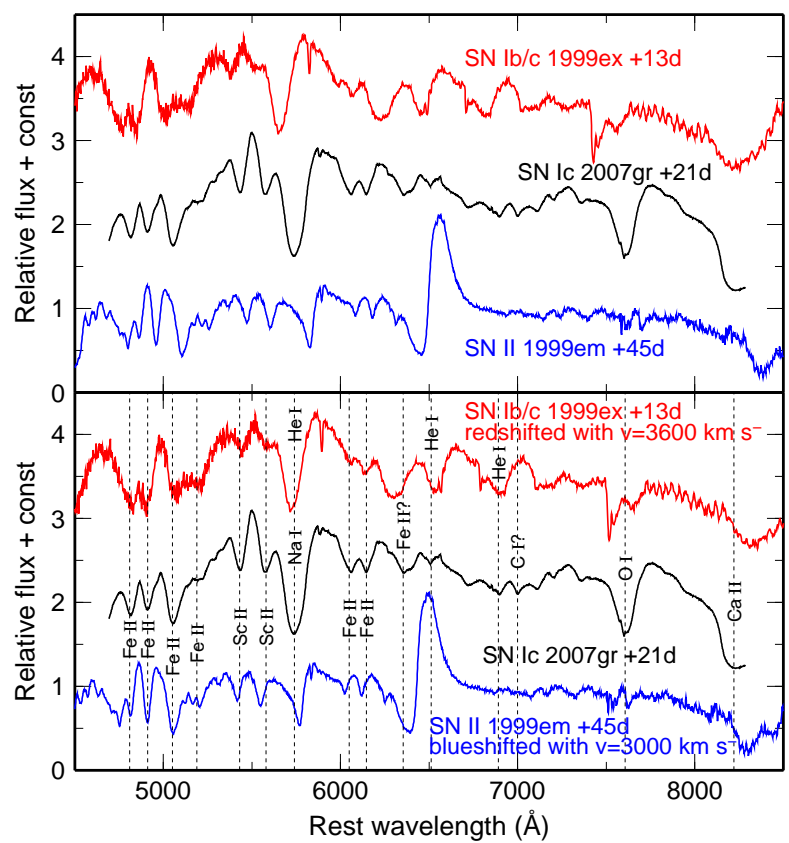

FIG. 1.- Upper: The spectrum of SN 2007gr (black) at 21 days after the maximum $(+21$ days $)$, compared with the spectrum of SN Ib/c 1999ex at +13 days (red, Hamuy et al. 2002) and of SN IIp $1999 \mathrm{em}$ at +45 days (blue, Leonard et al. 2001). The redshift of the host galaxy is corrected. Lower: Same as upper panel but the spectrum of SN $1999 \mathrm{ex}$ is redshifted by $v=3600 \mathrm{~km} \mathrm{~s}^{-1}$ and the spectrum of SN $1999 \mathrm{em}$ is blueshifted by $v=3000 \mathrm{~km} \mathrm{~s}^{-1}$.

ing the rise time of 16 days (Valenti et al. 2008).

We used a 0. . 8 width slit, a 300 lines $\mathrm{mm}^{-1}$ grism and SY47 order-cut filter, giving a resolution of $\lambda / \Delta \lambda \sim 650$. The linear polarimetric module of FOCAS consists of a rotating superachromatic half-wave plate and a crystal quartz Wollaston prism. Both the ordinary and extraordinary rays are recorded on the CCD simultaneously. With the four integrations at $0^{\circ}, 45 .^{\circ}, 22.5^{\circ}$ and $67.5^{\circ}$ position of the half-wave plate, Stokes $Q$ and $U$ were derived as in Tinbergen (1996). For the degree of polarization $P$, the polarization bias correction was done using the results of Patat \& Romaniello (2006). Total exposure time of four integrations was $1200 \mathrm{sec}$.

The flux was calibrated using the observation of the standard star G191B2B (Massey et al. 1988; Oke 1990). Telluric absorptions in the SN spectrum were removed using those in the standard star spectrum. Spectropolarimetry of this unpolarized star was also performed with the same configuration as for the SN observation. From this observation, it was confirmed that the instrumental polarization is less than $0.1 \%$. The position angle was calibrated by the observation of dome flats through the fully-polarizing filter with a known position angle. The wavelength dependence of the optical axis of halfwave plate was also corrected through this procedure.

To check the validity of the derived polarization spectrum, we generated artificial shifts of $\pm 4-8$ pix in the dispersion direction in one of four SN frames, and did the same reduction. This shift corresponds to the slit width $(0 ! .8 \sim 8$ pix $)$ and this amount of shift could possibly happen within the slit width. We confirmed that this shift does not alter the polarization features discussed in this paper.

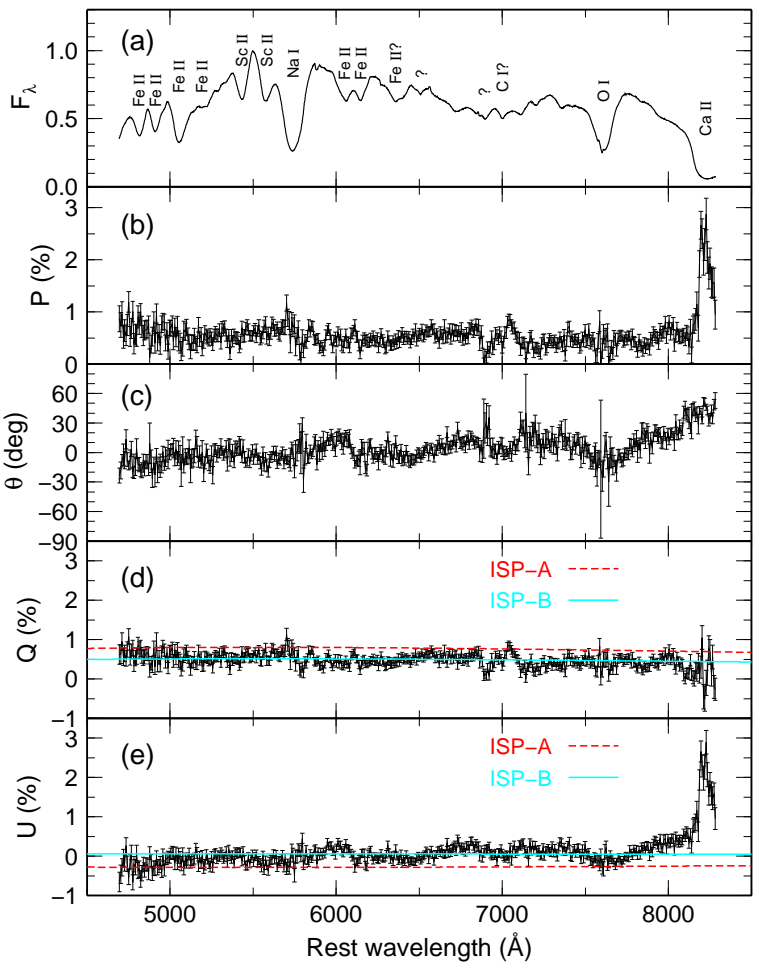

FIG. 2.- (a) The spectrum of SN $2007 \mathrm{gr}$ (in $10^{-14} \mathrm{erg} \mathrm{s}^{-1} \mathrm{~cm}^{-2}$ $\AA^{-1}$ ) at 21 days after the maximum, (b) the degree of polarization $P$, (c) polarization angle $\theta$, and (d, e) Stokes parameters $Q$ and $U$. Interstellar polarization (ISP) is not corrected in $P, Q$, $U$ and $\theta$. The polarization data are binned to $9 \AA$, similar to the spectral resolution. The red dashed line and cyan solid line show the two possible ISPs: $\left(p_{\max , \text { ISP-A }}, \theta_{\text {ISP-A }}\right)=\left(0.80 \%, 170^{\circ}\right)$ and $\left(p_{\text {max }, \text { ISP-B }}, \theta_{\text {ISP-B }}\right)=\left(0.51 \%, 3^{\circ}\right)$, respectively.

\section{RESULTS}

\subsection{Spectroscopic Properties}

The upper panel of Figure 1 shows the spectrum of SN 2007 gr (black) compared with SN 1999ex at +13 days from B maximum (red, Hamuy et al. 2002) and SN $1999 \mathrm{em}$ at +45 days from B maximum (blue, Leonard et al. 2001). These two SNe show most similar features to SN $2007 \mathrm{gr}$ at these epochs. The later epoch of Type II SN 1999em reflects the slower evolution of Type II SNe than Type Ib/c SNe. The redshift of the host galaxy is corrected.

If the spectrum of SN $1999 \mathrm{ex}$ is redshifted by $v=3600$ $\mathrm{km} \mathrm{s}^{-1}$ (lower panel), the positions of several absorption minima in SN 2007gr are similar to those in the shifted spectrum of SN $1999 \mathrm{ex}$, especially at the wavelength range redder than $6500 \AA$. Although there are weak troughs at the positions of the He I $\lambda 6678$ and 7065 in SN 2007gr, these are unlikely to be the He lines because of the absence of the He I $\lambda 20581$, which is usually stronger than the He I lines in the optical range (Valenti et al. 2008). Note that the absorption at $5750 \AA$ in SN 2007 gr should be mainly by $\mathrm{Na}$ I $\lambda 5889$.

Valenti et al. (2008) noted that SN 2007gr shows the $\mathrm{C}$ I and C II lines in the optical and NIR wavelength range. In this respect, SN $2007 \mathrm{gr}$ is a special class of Type Ic SNe, or "carbon-rich" Type Ic SN (Valenti et al. 2008). In our spectrum, one clear difference from the shifted spectrum of SN 1999 ex is seen at $7000 \AA$. SN 


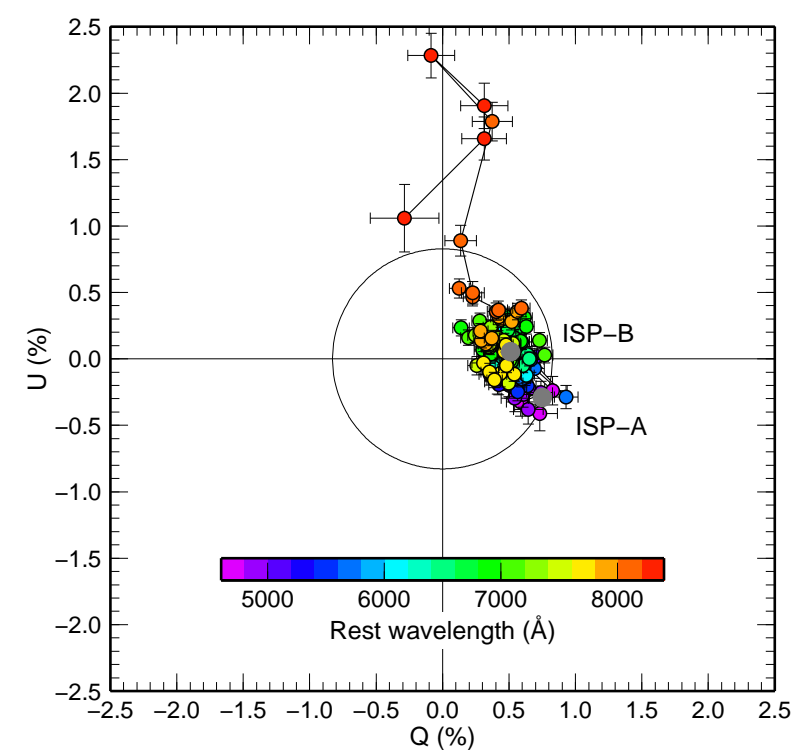

FIG. 3.- $Q-U$ diagram of the polarization data before the correction of interstellar polarization (ISP). Different colors show the wavelength as shown in the color scale. Possible two choices of ISP are marked with gray circles. The polarization data are binned to $25 \AA$. The thin large circle shows the upper limit of ISP $(0.83 \%)$.

2007gr has a weak absorption feature while SN 1999ex does not. This could be the $\mathrm{C}$ I $\lambda 7175$ line. The weak trough at $6500 \AA$ could be attributed to the C II $\lambda 6578$ line, but then the Doppler velocity of the line $(v \sim 3000$ $\left.\mathrm{km} \mathrm{s}^{-1}\right)$ is too small compared with other lines $(v \sim 7000$ $\left.\mathrm{km} \mathrm{s}^{-1}\right)$.

If the spectrum of $\mathrm{SN} 1999 \mathrm{em}$ is blueshifted by $v=$ $3000 \mathrm{~km} \mathrm{~s}^{-1}$, the spectral features are in good agreement with those in SN 2007gr especially at the wavelength range bluer than $6200 \AA$. These are mainly the absorptions of Na I, Sc II and Fe II (Leonard et al. 2001).

\subsection{Spectropolarimetric Properties}

Figure 2 shows the spectrum of SN 2007gr, the degree of polarization $\left(P \equiv \sqrt{Q^{2}+U^{2}}\right)$, polarization angle $[\theta \equiv 0.5 \operatorname{atan}(U / Q)$, measured from north to east], Stokes parameters $Q$ and $U$ (from upper to lower panels). Note that the interstellar polarization (ISP) is not corrected in Figure 2.

The degree of polarization $P$ is $\sim 0.3-0.5 \%$ at 4600 $8000 \AA$ (Fig. 2). Although the change in the degree of polarization at this wavelength range is not significant, the polarization angle increases with the wavelength, caused by the decreasing and increasing trend in Stokes $Q$ and $U$, respectively. This does not directly imply that the SN has intrinsic polarization with the increasing position angle with wavelength since the data still suffer from ISP.

The properties of the spectropolarimetric data are more comprehensive in the $Q-U$ diagram (Fig. 3). In Figure 3, Stokes parameters at the different wavelength are shown in different colors, representing its wavelength as in the color scale below. The majority of the data except for the reddest part is aligned in straight line, although it is not very prominent. The similar alignment in the $Q-U$ diagram has been observed in many core-collapse SNe (e.g., Wang et al. 2001; Leonard et al. 2002b).

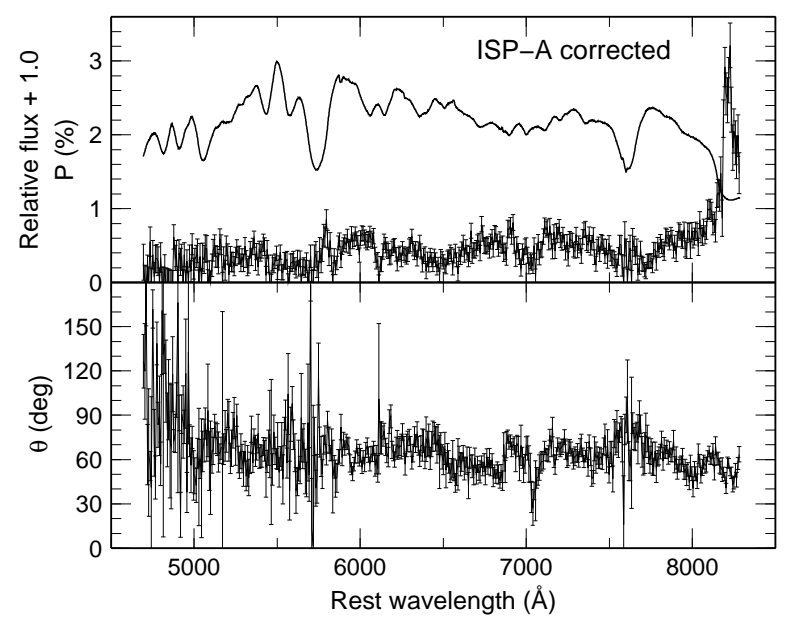

FIG. 4.- The polarization spectrum (upper) and position angle (lower) of SN $2007 \mathrm{gr}$ corrected with ISP-A. ISP-A is ( $p_{\text {max,ISP-A, }}$ $\left.\theta_{\text {ISP-A }}\right)=\left(0.80 \%, 170^{\circ}\right)$. The polarization data are binned to $9 \AA$.

The Ca II IR triplet has a large degree of polarization, reaching $\sim 3 \%$. The polarization is largest at the absorption minimum (Fig. 2). The large polarization level of Ca II is independent on the choice of ISP (§3.3).

No other line shows as strong polarization as in the Ca II line. We see, however, a small peak of polarization at $5750 \AA$ (near the Na I line) and a fluctuation around $7000 \AA$ (near the possible C I line). Note that the intrinsic polarization of SN at these lines depends on the choice of ISP (§3.3).

\subsection{Interstellar and Intrinsic Polarization}

In order to discuss the properties of SN, the contribution of ISP in the line of sight to the SN position should be removed from the observed data. The amount of reddening gives the upper limit of ISP. The total reddening is $E(B-V)_{\text {tot }}=0.092$, which is the sum of the Galactic reddening $E(B-V)_{\text {Gal }}=0.062$ and the host reddening $E(B-V)_{\text {host }}=0.03$ (Valenti et al. 2008). Then, the maximum degree of ISP is $p \sim 0.83 \%$, using the relation of $p / E(B-V)<9 \%$ (Serkowski et al. 1975). This maximum degree of ISP is shown in the circle in Figure 3.

To estimate the ISP, we should use SN data themselves unless detailed studies on ISP in this line of sight are available. With SN data, ISP could be estimated by the following methods (1) - (4); (1) using polarimetry of SN at late epochs, when the SN ejecta become thin and electron scattering is not effective, (2) following the time evolution of SN polarimetry and assuming the time-independent component as ISP (e.g., Leonard et al. 2002b), (3) taking the polarization level at the strong PCygni emission peak, where complete depolarization can be expected (e.g., Kawabata et al. 2002; Maund et al. 2007a), and (4) assuming SN photosphere has a single polarization angle, i.e., an axisymmetric structure (e.g., Wang et al. 2003b).

The methods (1) and (2) are rather robust, but unfortunately these cannot be used for our one-epoch data. The method (3) can be taken even in one-epoch data. However, since the emission peak of the most polarized Ca II IR triplet is not covered, this approach is also not 


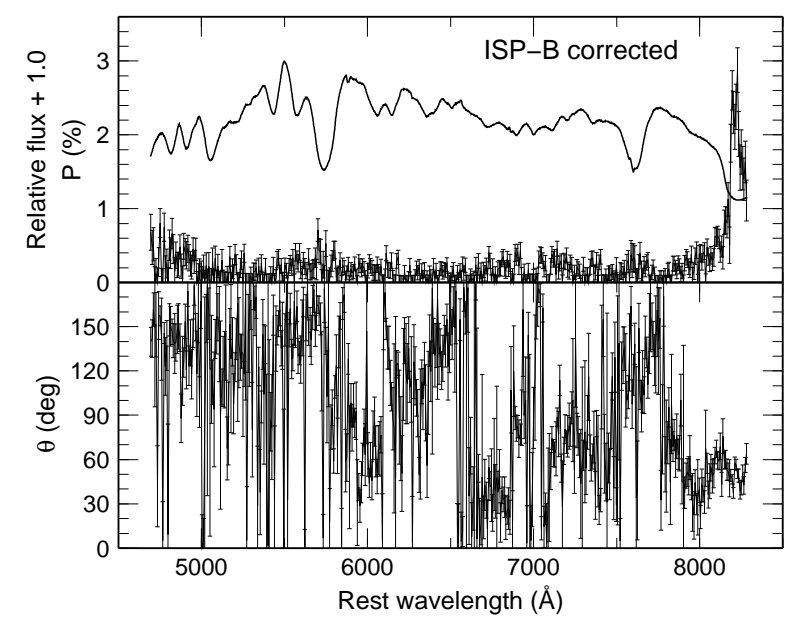

FIG. 5.- Same as Fig. 4, but polarization data are corrected assuming ISP-B $\left(p_{\max , \text { ISP-B }}, \theta_{\text {ISP-B }}\right)=\left(0.51 \%, 3^{\circ}\right)$. The spectra are binned to $9 \AA$.

effective for our data. Although the method (4) relies on the SN theory, it can be applied to our SN 2007gr data because the polarization data are aligned in the straight line in the $Q-U$ diagram (Fig. 3). It should be emphasized, however, that this method is less reliable compared with the other three methods.

Adopting the method (4), we could take both sides of the straight line in the $Q-U$ diagram as an estimated ISP. Since it is expected that the bluer spectrum suffers from more depolarization (e.g., Howell et al. 2001; Wang et al. 2003b), it is a sound assumption to take the bluest side as ISP. The estimated ISP (ISP-A) is ( $p_{\text {max,ISP-A }}$, $\left.\theta_{\text {ISP-A }}\right)=\left(0.80 \%, 170^{\circ}\right)$ as shown in the gray circle in Figure 3 . Here, $p_{\max }$ is the degree of ISP at $\lambda_{\max }$. We assume $\lambda_{\max }=5500 \AA$, which is the wavelength of the maximum ISP (Serkowski et al. 1975). The wavelength dependence of ISP is taken from Serkowski et al. (1975);

$$
p(\lambda)=p_{\max } \exp \left[-K \ln ^{2}\left(\frac{\lambda_{\max }}{\lambda}\right)\right],
$$

which is commonly accepted for ISP in our galaxy. Here $K$ is given as $K=0.01+1.66 \lambda_{\max }(\mu \mathrm{m})=0.932$ (Whittet et al. 1992). The $Q$ and $U$ components of ISP-A are shown in the red dashed line in Figure $2^{7}$.

The polarization data after the subtraction of ISP-A are shown in Figure 4. The polarization angle $(\theta)$ is $\sim$ $40-80^{\circ}$ for a wide range of the spectrum, although there is a large scatter in the blue wavelength. The polarization angle in the line-free region at $6600-6700 \AA$ is $60 \pm 5^{\circ}$. The degree of polarization is $0.3-0.7 \%$ except for the reddest part, and it is $\sim 0.5 \pm 0.2 \%$ at $6600-6700 \AA$.

It should be noted that there are cautions when we take ISP-A. Since the reddening neither in our Galaxy nor the host galaxy is negligible (Valenti et al. 2008), it is expected that the light from SN suffers from ISP both in our Galaxy $(\lesssim 0.56 \%)$ and the host galaxy $(\lesssim 0.27 \%)$. Thus, the choice of ISP-A $\left(p_{\max , \text { ISP-A }}=0.8 \%\right)$, being

\footnotetext{
7 It should be noted that the wavelength dependence of ISP in the host galaxy could be different from Serkowski's law. If the same wavelength dependence can be assumed, it is not a bad approximation to use the wavelength dependence of Serkowski's law for the sum of ISPs in our Galaxy and the host galaxy because ISP-A is close to the maximum degree of ISP.
}

close to the upper limit of ISP $\left(p_{\max }=0.83 \%\right)$, requires a fine alignment of the position angles of ISP, which could be unlikely. In addition, although the position angle of ISP in the host galaxy is expected to be aligned with the spiral arm $\left(\theta \sim 30^{\circ}\right.$ in this case, see e.g., Scarrott, Ward-Thompson, \& Warren-Smith 1987; Scarrott et al. 1993 ), the position angle of ISP-A is displaced by $\sim 40^{\circ}$ from that of the spiral arm.

As an alternative choice of ISP, we take ISP-B at the center of polarization data in the $Q-U$ diagram, $\left(p_{\text {max }, \text { ISP-B }}, \theta_{\text {ISP-B }}\right)=\left(0.51 \%, 3^{\circ}\right)$, which is shown in the gray circle in Figure 3. The $Q$ and $U$ components of this ISP are shown in Figure 2 in the cyan solid line 8 . Figure 5 shows the polarization after the correction of ISP-B. Since the straight line in the $Q-U$ diagram is not very prominent, this choice results in the small degree of intrinsic polarization at a wide wavelength range except for the Ca II IR triplet (upper panel). Although the polarization angle is very scattered due to the small $Q$ and $U$, a large portion of the data has $\theta \sim 150^{\circ}$ and $\sim 60^{\circ}$ at mainly blue and red wavelength range, respectively.

Both ISP-A and ISP-B are not inconsistent with the polarization degrees and angles of field stars in the stellar polarization catalog of Heiles (2000) close to the SN position. Thus, with the available data only, we cannot distinguish the two possibilities of ISP. We thus discuss possible interpretations of the polarization data with these two ISPs.

\section{GEOMETRY OF SN $2007 \mathrm{gr}$}

In the following sections, we discuss the multidimensional ejecta structure of SN 2007gr. With the continuum polarization, we can infer the asymmetry of electron scattering-dominated photosphere (e.g., Shapiro \& Sutherland 1982; Höflich 1991). In addition, with the polarization properties across the P-Cygni profiles, we can discuss the distribution of elements. In strong PCygni profiles, the polarization often has maximum (minimum) at the minimum (maximum) of the flux, which is so called "inverted P-Cygni" profile (Jeffery 1989, 1991). The polarization level becomes maximum at the absorption minimum because the lights that are scattered through smallest angles by electrons (and thus have less polarization) are effectively scattered out by the line at the absorption minimum. The polarization minimum at the emission peak is caused by the dilution due to the directly-coming, unpolarized emission by the line.

In a simple ellipsoidal case, where only the unpolarized or polarization-cancelled lights are scattered by the line, the polarization at the absorption trough $\left(p_{\text {trough }}\right)$ is limited as

$$
p_{\text {trough }} \lesssim p_{\text {cont }} \frac{I_{\text {cont }}}{I_{\text {trough }}},
$$

where $p_{\text {cont }}, I_{\text {cont }}$, and $I_{\text {trough }}$ are the continuum polarization, the continuum flux, and the flux at the trough, respectively (Leonard et al. 2001). If the line polarization larger than the limit of Eq. (2) is observed at the trough, it means that the simple ellipsoidal model does

\footnotetext{
${ }^{8}$ We simply assume Serkowski's law also in ISP-B, meaning the alignment of position angle of ISP in our Galaxy and host galaxy, which is not necessarily required. But the wavelength dependence of ISP is very weak and this treatment is essentially similar to the subtraction of a wavelength-independent ISP.
} 


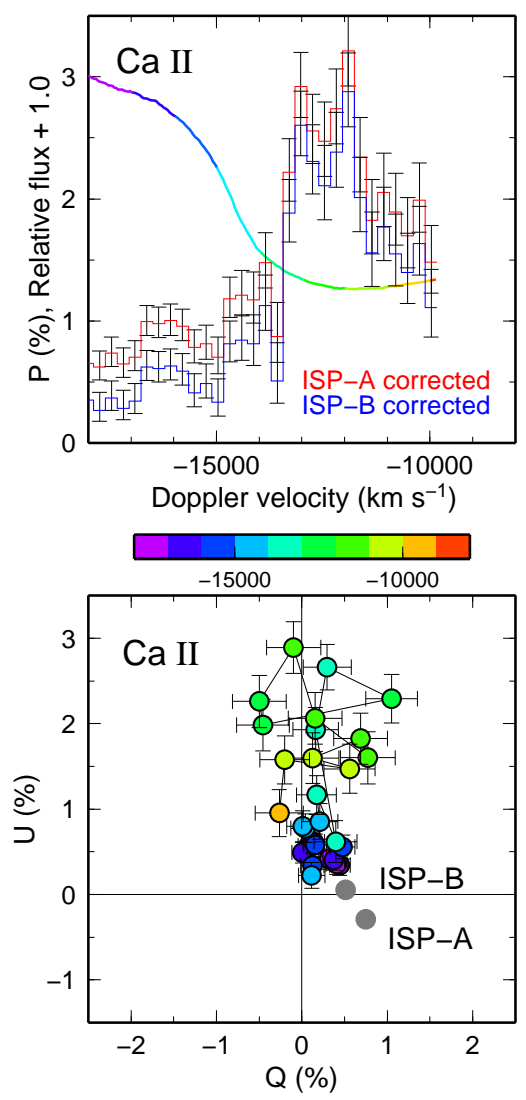

FIG. 6.- Polarization properties across the Ca II IR triplet (mean $\lambda 8567)$ line. Upper panels: The spectrum (color line) and the degree of the polarization. The red and blue lines show the polarization corrected with ISP-A and ISP-B, respectively. They are shown against the Doppler velocity measured from the rest wavelength. Lower panels: ISP-uncorrected $Q-U$ diagrams of the $\mathrm{Ca}$ II line. The ISP-A and ISP-B are shown in by the gray points. The color of each point shows the Doppler velocity. The spectra are binned to $9 \AA$.

not work and that the clumpy or patchy line forming region obscures a certain part of the photosphere, enhancing the polarization level.

In the upper panel of Figure 6, the flux spectrum and the ISP-corrected polarization spectrum versus Doppler velocity are shown near the Ca II IR triplet (mean $\lambda 8567)$. Figure 7 is the same as Figure 6 but for the O I $\lambda 7774, \mathrm{Na}$ I $\lambda 5889$, possible $\mathrm{C}$ I $\lambda 7175$ lines. The red and blue lines show the ISP-A and ISP-B corrected polarization, respectively. In the lower panels of Figures 6 and 7, the $Q-U$ diagrams at these lines are shown. Since the change of ISP across the line is negligible, ISP is not corrected for in the $Q-U$ diagram, where the two choices of ISP are shown by the gray circles. The color in these plots shows the Doppler velocity. The spectra in Figures 6 and 7 are binned to $9 \AA$.

\subsection{Interstellar Polarization-A: Axisymmetric Photosphere}

Adopting ISP-A, the intrinsic SN polarization has an almost constant position angle (Fig. 4). If we take the line free region (6600-6700 $\AA$ ) as the continuum region, the continuum has the polarization of $\sim 0.5 \%$, with the position angle $\theta \sim 60^{\circ}$. This suggests that the shape of the SN photosphere projected to the sky is deformed at a level of $\sim 10 \%$ (e.g., Höflich 1991). The major axis of the ellipsoid is located in the direction of $\sim 150^{\circ}$ measured from north to east (i.e., $\sim 30^{\circ}$ from north to west) because the polarization is orthogonal to the radial direction of the projected photosphere (see A1 and A2 Fig. 8).

\subsubsection{Calcium}

The polarization across the Ca II line is prominent, reaching $\sim 3 \%$ at the absorption minimum as seen from the red line in the upper left panel of Figure 6 . The polarization angle of the Ca II line is $\sim 50^{\circ}$, which is close to that of the continuum $\left(\sim 60^{\circ}\right)$.

At the Ca II line in SN 2007gr, $I_{\text {cont }} / I_{\text {trough }} \sim 5-6.5$ given the uncertainty of the continuum level. Taking the continuum polarization is $p_{\text {cont }} \sim 0.5 \%$, Eq. (2) gives the limit of $p \lesssim 2.5-3.25 \%$. Thus, although the simple ellipsoidal model could be accepted, a selective obscuration enhancing the uncancelled polarization component is preferred to robustly explain the observed high polarization level at the line $(\sim 3 \%)$, considering the idealized situation assumed in Eq. (2). Since the position angle of the Ca II line is similar to that of the continuum, the Ca II line forming region should selectively obscure the light originated around the minor axis of the SN photosphere (see A1 and A2 of Fig. 8).

\subsubsection{Oxygen, Sodium and Carbon}

Although the absorption line of O I is strong, no significant change of polarization is observed across the line (the left panel of Fig. 7 ). At this line, $I_{\text {cont }} / I_{\text {trough }} \sim 2$, and thus, polarization of $\sim 1 \%$ at the absorption minimum can be expected from Eq. (2). This means that the $\mathrm{O}$ I line obscures not only unpolarized or polarizationcancelled light but also some part of the projected photosphere near its major axis. Thus, the distribution of $\mathrm{O}$ I follows the photosphere, being clearly different from that of Ca II.

The polarization at the $\mathrm{Na}$ I line is marginally enhanced (the center panel in Fig 6), although the maximum of polarization is not located exactly at the absorption minimum of the flux. Note that this behavior depends on the choice of ISP. Overall, the line does not have significant polarization as expected from Eq. (2), suggesting a similar distribution of $\mathrm{Na}$ I to $\mathrm{O}$ I.

There is no large change of the polarization at the possible C I line (the right panel of Fig. 7). However, this is just a natural consequence of the weakness of the line (i.e., $I_{\text {cont }} / I_{\text {trough }} \sim 1$ ).

\subsubsection{Geometry of the Ejecta}

We summarize the polarization properties under the assumption of ISP-A. The continuum polarization suggests that there is a mild asphericity in the photosphere. The distribution of $\mathrm{O}$ I and $\mathrm{Na}$ I follows the photosphere. On the other hand, the distribution of Ca II is different, which is likely to obscure the photosphere near its minor axis. This effectively enhances the polarization level, unchanging the polarization angle. There is not much information on the distribution of $\mathrm{C}$ I.

If the photosphere has a prolate shape, the observed properties might be consistent with the bipolar explosion seen from near the equatorial plane (A1 of Fig. 8). The 

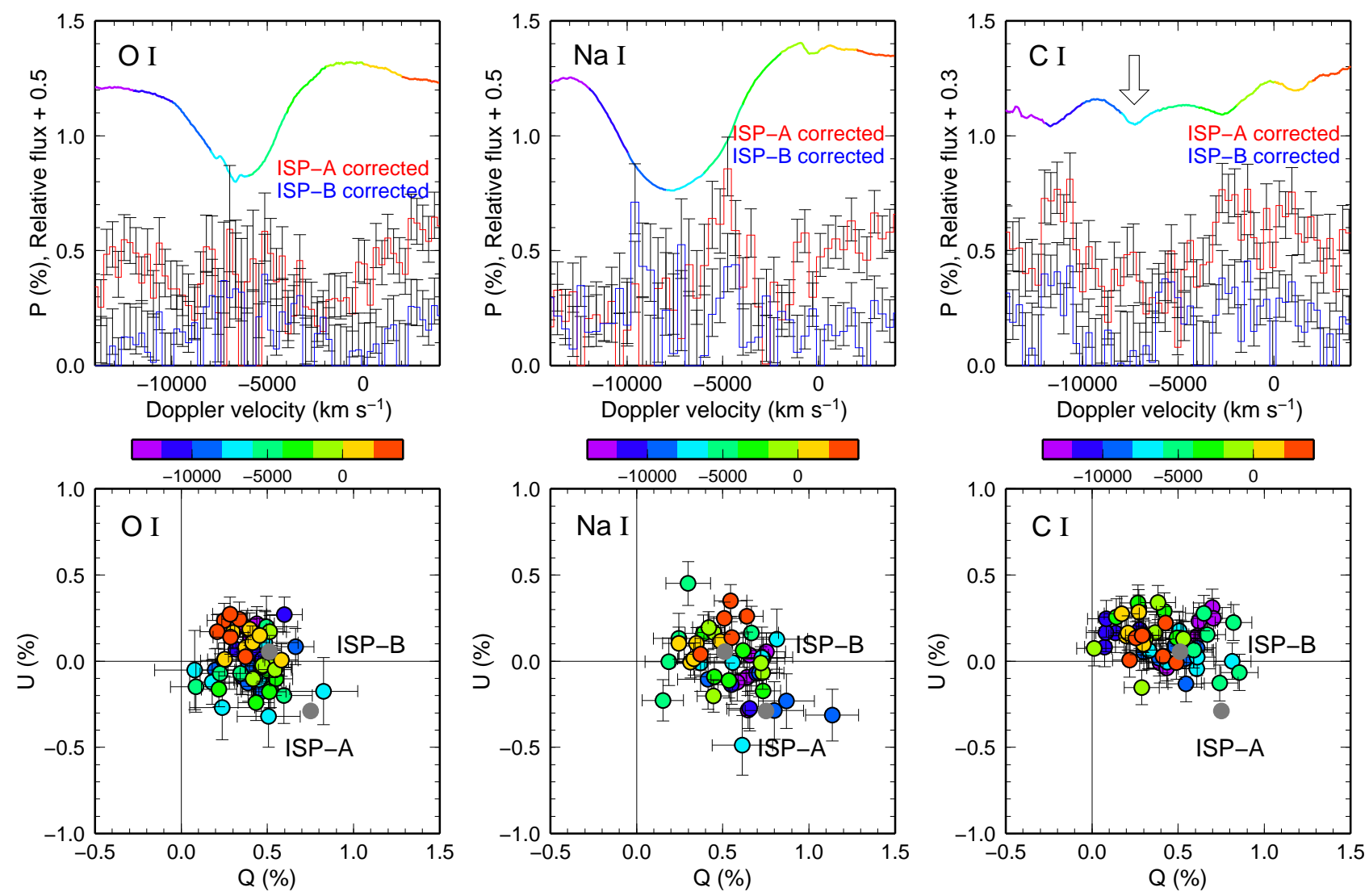

Fig. 7.- Polarization properties across the $\mathrm{O}$ I $\lambda 7774, \mathrm{Na}$ I $\lambda 5889$, and possible $\mathrm{C}$ I $\lambda 7175$ lines (from left to right). Upper panels: The spectrum (color line) and the degree of the polarization. The red and blue lines show the polarization corrected with ISP-A and ISP-B, respectively. They are shown against the Doppler velocity measured from the rest wavelength. Lower panels: ISP-uncorrected $Q-U$ diagrams of the Ca II line. The ISP-A and ISP-B are shown in by the gray points. The color of each point shows the Doppler velocity. The spectra are binned to $9 \AA$.

pole points to $\sim 30^{\circ}$ from north to west in the sky. In the bipolar explosion (e.g., Khokhlov et al. 1999; Maeda et al. 2002), the heavy element including ${ }^{56} \mathrm{Ni}$ is synthesized in the polar region. In such a model, the polar region is more ionized and a prolate photosphere can be formed as suggested by Höflich (2001) for Type II SNe. Further, a disk-like structure remains in the equatorial plane and $\mathrm{Ca}$ II can also be enhanced in the disk due to the low temperature (Tanaka et al. 2007). However, in this scenario, the distribution of $\mathrm{Na}$ I and $\mathrm{O}$ I may follow Ca II, which is not consistent with the observation.

Alternatively, in the bipolar explosion, the photosphere could have an oblate shape, tracing the dense disk-like region. This may be true at $\gtrsim 30$ days after the explosion (Maeda et al. 2006). Then the observed continuum polarization requires that the explosion is seen from the slightly off-axis direction (A2 of Fig. 8). In this case, explosively synthesized $\mathrm{Ca}$ near the polar region can make the Ca II absorption. In addition, the distribution of $\mathrm{O}$ I and Na I may follow the photosphere (Tanaka et al. 2007). This ejecta structure is qualitatively consistent with the observed properties.

\subsection{Interstellar Polarization-B: Almost Spherical Photosphere}

If we take ISP-B, the polarization is very small at a wide wavelength range (Fig. 5). The photosphere has a possible two-axis structure with the position angles of $\sim 150^{\circ}$ and $60^{\circ}$ at the blue and red spectra, respectively, although it is quite marginal given the small polarization level (lower panel of Fig. 5).

Small continuum polarization suggests an almost spherical photosphere. The small continuum polarization could be realized more easily when the radial density profile is steeper because of the smaller contribution of the orthogonally-scattered, and thus more-polarized lights. This is consistent with the suggestion by Valenti et al. (2008): the steep density profile is required to explain the slow recession of the photosphere.

If there is little continuum polarization, from Eq. (2), any large line polarization undoubtedly suggests an aspherical distribution of the ion, which can cause the selective obscuration of the photosphere. This is similar to what is usually found in Type Ia SNe (e.g., Wang et al. 2003a; Leonard et al. 2005b).

In fact, with the assumption of ISP-B, a strong polarization is seen in the Ca II line. This requires an asymmetric distribution of $\mathrm{Ca}$ II, and the clump(s) or disk-like distribution may be possible.

There is no conspicuous polarization change across the $\mathrm{O}$ I, Na I and $\mathrm{C}$ I line. This suggests that these elements are distributed almost spherically, similar to the photosphere.

In summary, the SN polarization with ISP-B can be explained by the almost spherical photosphere. Only the distribution of $\mathrm{Ca}$ II is largely deviated from spherical 
TABLE 1

Comparison of Spectropolarimetric Features

\begin{tabular}{|c|c|c|c|c|c|c|c|}
\hline SN name Ref. & Type & Epoch $^{\mathrm{a}}$ & $p_{\text {cont }}{ }^{\mathrm{b}}$ & $\Delta p_{\mathrm{Ca}}{ }^{\mathrm{c}}$ & $\Delta p_{\mathrm{O}}{ }^{\mathrm{d}}$ & $\Delta p_{\mathrm{Na}}{ }^{\mathrm{e}}$ & $\Delta p_{\mathrm{Fe}}{ }^{\mathrm{f}}$ \\
\hline SN 2007gr (ISP-A) & Ic & 37 & $\sim 0.5$ & 2.5 & 0 & 0 & 0 \\
\hline SN 2007gr (ISP-B) & & & $\lesssim 0.2$ & 2.5 & 0 & 0 & 0 \\
\hline \multirow[t]{2}{*}{ SN 2002ap $^{1}$} & broad Ic & $11-16$ & $\sim 0.5$ & $\lesssim 0.2$ & 0.4 & - & - \\
\hline & & $37-41$ & $\sim 0.5-1$ & 1.5 & 0.2 & - & - \\
\hline SN 2005bf ${ }^{2}$ & pec. Ib & 34 & $\lesssim 0.45$ & 3.5 & - & - & 1 \\
\hline SN 2003gf 3 & Ic & 30 & $\approx 0.5$ & 1.5 & 1.3 & 0.4 & $0.5(?)$ \\
\hline \multirow[t]{2}{*}{ SN 2004dj ${ }^{4}$} & IIp & 39 & 0 & 0 & 0 & 0 & 0 \\
\hline & & 91 & 0.6 & 0 & - & 0.7 & 0 \\
\hline $\mathrm{SN} 1987 \mathrm{~A}^{5}$ & pec. II & 100 & $\sim 0.5$ & 3 & - & -0.2 & - \\
\hline
\end{tabular}

References. - (1) Kawabata et al. (2002); Leonard et al. (2002b); Wang et al. (2003b), (2) Maund et al. (2007), (3) Leonard \& Filippenko (2005), (4) Leonard et al. (2006), (5) Cropper et al. (1988); Jeffery (1991)

${ }^{\text {a}}$ Estimated days after the explosion

${ }^{\mathrm{b}}$ Continuum polarization $(\%)$

${ }^{\mathrm{c}}$ Polarization change at $\mathrm{Ca}$ II line trough $(\%)$

${ }^{\mathrm{d}}$ Polarization change at $\mathrm{O}$ I line trough $(\%)$

ePolarization change at $\mathrm{Na}$ I line trough (\%)

${ }^{\mathrm{f}}$ Polarization change at Fe II line trough (\%)

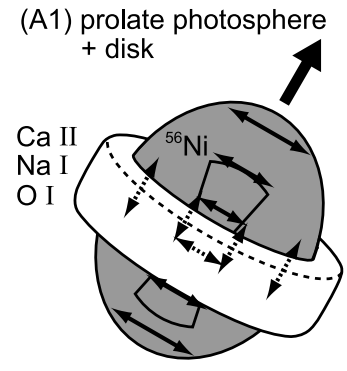

(A2) oblate photosphere + clump

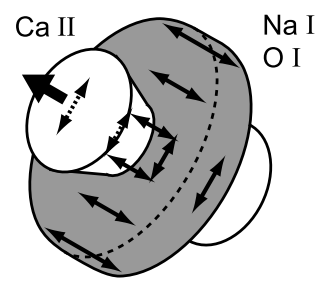

(B) spherical photosphere + clump
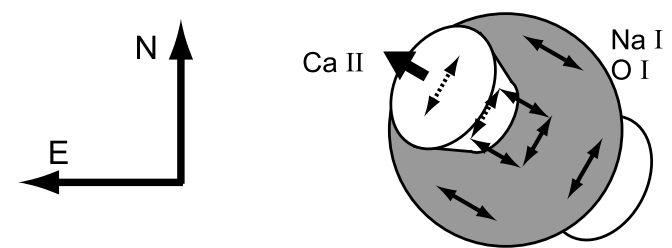

FIG. 8.- Schematic illustrations of possible bipolar explosion models. Shaded area shows the photosphere. The thin arrows represent the polarization vector. Photons scattered-out by the line forming region (unshaded area) are shown in dashed arrows. The thick arrows show the polar direction. A1 and A2 are the models with ISP-A while B is with ISP-B. The degree of asphericity is exaggerated. Both A1 and A2 would show continuum polarization with the position angle of $\sim 60^{\circ}$. The strong line polarization at the $\mathrm{Ca}$ II line with similar position angle can also be explained. However, case A1 predicts similarly strong line polarization at the $\mathrm{O}$ I and $\mathrm{Na}$ I lines. For case A2, $\mathrm{O}$ I and $\mathrm{Na}$ I may follow the photosphere, which is consistent with the observed polarization. Case $\mathrm{B}$ does not have continuum polarization but has line polarization at the Ca II line with the position angle of $\sim 60^{\circ}$.

symmetry, and other ions such as $\mathrm{O}$ I, Na I, and $\mathrm{C}$ I are distributed almost spherically.

Constraints on the explosion models are not strong. In the bipolar explosion models, $\mathrm{Ca}$ in the pre-explosion star could be enhanced in disk-like region (see A1 of Fig. 8) due to the high density and the low ionization there. However, as discussed in $\S 4.1$, this may not be consistent with the observed properties because $\mathrm{O}$ and $\mathrm{Na}$ are also abundant before the explosion, i.e., O I and Na I may also have the disk like distribution.

Alternatively, the explosively synthesized Ca II distributed near the axis ( $\mathrm{B}$ in Fig. 8) can explain the observation. This may be realized by less-aspherical explosion than case A2 in Figure 8.

\subsection{Comparison with Other Core-Collapse SNe}

There are only a small number of spectropolarimetric observations of Type Ib/c SNe to date (SN Ic 1997X; Wang et al. 2004, SN Ic 1997ef; Wang et al. 2004, SN Ic 1998bw; Patat et al. 2001, SN Ic 2002ap; Kawabata et al. 2002; Leonard et al. 2002b; Wang et al. 2003b, SN Ic 2003dh; Kawabata et al. 2003, SN Ic 2003gf; Leonard \& Filippenko 2005a, SN Ib 2005bf; Maund et al. 2007a; SN Ic 2006aj; Maund et al. 2007b). Note that SNe 1997ef, 1998bw, 2002ap, 2003dh, 2006aj are all broad-line Type Ic SNe, among which SNe 1998bw and 2003dh are estimated to have an explosion energy exceeding $10^{52} \mathrm{erg}$, thus being called hypernovae (e.g., Nomoto et al. 2007). Thus, SN 2007gr is one of the rare spectropolarimetric sample of normal Type Ib/c SNe.

We first compare the results with SN 2002ap, best observed Type Ic SN in spectropolarimetry (Kawabata et al. 2002; Leonard et al. 2002b; Wang et al. 2003b). There are also fair similarities between ISP-A subtracted SN 2007gr at 21 days after the maximum ( 37 days after the explosion) and SN 2002ap at 30 days after the maximum ( $\sim 41$ days after the explosion, assuming the rise time of 11 days, Mazzali et al. 2002): (1) a moderate continuum polarization level $(\sim 0.5 \%)$, and $(2)$ a strong polarization in the Ca II IR triplet with the similar position angle to that of the continuum (see March 2002 data in Kawabata et al. 2002; Leonard et al. 2002b). Note that SN 2002ap shows the two distinct polarization angle in continuum and the $\mathrm{Ca}-\mathrm{O}$ absorption at maximum epoch.

The light curve of SN 2007gr is similar to SN 2002ap but the spectra of SN 2007gr show narrower lines than in SN 2002ap (Valenti et al. 2008). Valenti et al. (2008) speculated that SN 2007gr and SN 2002ap are the bipolar 
explosions observed from the minor and major axis, respectively. Our data do not support this hypothesis since there are similarities in polarization data of two objects and the polarization of SN $2007 \mathrm{gr}$ is likely to be interpreted by the viewing angle near the major axis $(\S 4.1$ and $\S 4.2)$.

Another comparison can be made with the peculiar SN Ib 2005bf (Anupama et al. 2005; Tominaga et al. 2005; Folatelli et al. 2006; Maeda et al. 2007). Maund et al. (2007) showed spectropolarimetric data of SN 2005bf at 6 days before the 2 nd maximum, which is $\sim 34$ days after the explosion (assuming the rise time of $\sim 40$ days, Tominaga et al. 2005). SN 2005bf also showed a continuum polarization $(\sim 0.45 \%)$ and a large polarization level across the Ca II triplet with the polarization angle almost aligned to that of the photosphere. In SN 2005bf, however, there are distinct polarization features at the Fe II lines, which are not seen in SN 2007gr. This may be due to the difference in the degree of asphericity, the line of sight, or the epoch of the observation, i.e., difference in the observed layer.

The comparison with Type II SNe after the plateau phase is also useful. In Type II SNe, the increase of polarization degree after the plateau phase have been observed (e.g., Leonard et al. 2001, 2006). At this epoch, the He-core is exposed, which would be a similar to an exploding $\mathrm{He} / \mathrm{C}+\mathrm{O}$ star (i.e., Type $\mathrm{Ib} / \mathrm{c} \mathrm{SNe}$ ).

In SN 2004dj (Leonard et al. 2006), the continuum polarization is $\sim 0.6 \%$ after the plateau phase, which is similar to that of SN 2007gr. Large line polarization changes are observed at the $\mathrm{Na}$, $\mathrm{H} \alpha$ and $\mathrm{Ca}$ II lines. At the latter two lines, the P-Cygni emission component is much stronger than the absorption and only the depolarization is observed. At the $\mathrm{Na}$ I line, polarization change at a level of $\sim 0.7 \%$ is observed, which is not seen in SN $2007 \mathrm{gr}$. In SN 1987A, on the other hand, a small depolarization at the $\mathrm{Na}$ I line and a large polarization change $\sim 3 \%$ at the Ca II IR triplet are observed (Cropper et al. 1988; Jeffery 1991).

The polarization properties discussed above are summarized in Table 1

\section{CONCLUSIONS}

We have presented optical spectropolarimetric observations of Type Ic SN 2007gr at 21 days after maximum brightness ( $\sim 37$ days after the explosion). A strong polarization feature is observed across the Ca II IR triplet line, which is as high as $\sim 3 \%$ independently of the choice of ISP.

If we assume that the $\mathrm{SN}$ photosphere has a single polarization angle, the intrinsic polarization of the SN continuum is $\sim 0.5 \%$. This suggests that the axis ratio of the photosphere projected on the sky is different from unity by $\sim 10 \%$. The polarization angle of the continuum is $\sim 60^{\circ}$, which is similar to that of the strong polarization in the Ca II line $\left(\sim 50^{\circ}\right)$. The polarization of the continuum and the Ca II line in SN 2007gr are similar to those found in broad-line SN Ic 2002ap after maximum but not similar to SN 2002ap around maximum.

The spectropolarimetric features of SN 2007gr can be explained by a bipolar explosion viewed from the slightly off-axis direction (A2 in Fig. 8). The photosphere has an oblate shape in the equatorial plane. The explosively synthesized $\mathrm{Ca}$ II is distributed near the polar region, and it partially obscure the photosphere. The distributions of $\mathrm{O}$ I and $\mathrm{Na}$ I follow the photosphere, being different from Ca II. Given the uncertainty of ISP, however, the polarization data could also be interpreted by almost spherically symmetric photosphere and the aspherical Ca II distribution (B in Fig. 8).

We would like to thank the anonymous referee for valuable comments. M. T. would like to thank G. C. Anupama, D. K. Sahu, Daisuke Kato, and Masafumi Shimada for helpful suggestions and discussions. M. T. is supported by the JSPS (Japan Society for the Promotion of Science) Research Fellowship for Young Scientists. This research has been supported in part by World Premier International Research Center Initiative (WPI Initiative), MEXT, Japan, and by the Grant-in-Aid for Scientific Research of the JSPS (18104003, 18540231, 20540226) and MEXT (19047004, 20040004).

\section{REFERENCES}

Anupama, G. C., Sahu, D. K., Deng, J., Nomoto, K., Tominaga, N., Tanaka, M., Mazzali, P. A., \& Prabhu, T. P. 2005, 631, 125 Blondin, J. M., Mezzacappa, A., \& DeMarino, C., 2003, ApJ, 584, 971

Buras, R., Rampp, M., Janka, H.-Th., \& Kifonidis, K. 2006, A\&A, 447, 1049

Burrows, A., Livne, E., Dessart, L., Ott, C.D., \& Murphy, J. 2006 ApJ, 640, 878

Crockett R. M., et al. 2008, ApJ, 672, L99

Cropper, M., Bailey, J., McCowage, J., Cannon, R. D., \& Couch, W. J. 1988, MNRAS, 231, 695

Folatelli, G., et al. 2006, ApJ, 641, 1039

Hamuy, M., et al. 2002, AJ, 124, 417

Heiles, C. 2000, AJ, 119, 923

Höflich, P. 1991, A\&A, 246, 481

Höflich, P., Khokhlov, A., \& Wang, L. 2001, in AIP Conf. Proc. 586, 20th Texas Symposium on Relativistic Astrophysics, ed. J. C. Wheeler \& H.Martel (New York: AIP), 459

Howell, D. A., Höflich, P., Wang, L., \& Wheeler, J. C. 2001, ApJ, 556,302

Hwang, U., et al. 2004, ApJ, 615, L117

Jeffery, D. J. 1989, ApJS, 71, 951

Jeffery, D. J. 1991, ApJS, 77, 405

Kashikawa, N., et al. 2002, PASJ, 54, 819

Kawabata, K. S., et al. 2002, ApJ, 580, L39

Kawabata, K. S., et al. 2003, ApJ, 593, L19

Khokhlov, A. M., Höflich, P. A., Oran, E. S., Wheeler, J. C., Wang, L., \& Chtchelkanova, A. Y. 1999, ApJ, 524, L107

Kotake, K., Sawai, H., Yamada, S., \& Sato, K. 2004, ApJ, 608, 391
Leonard, D. C. Filippenko, A. V., Ardila, D. R., \& Brotherton, M. S. 2001, 553, 861

Leonard, D. C., et al. 2002a, PASP, 114, 35

Leonard, D. C., Filippenko, A. V., Chornock, R., \& Foley, R. 2002b, PASP, 114, 1333

Leonard, D. C., \& Filippenko, A. V. 2005, 1604-2004: Supernovae as Cosmological Lighthouses, ASP Conference Series, Vol. 342, Ed. by M. Turatto, et al., 330

Leonard, D. C., Li, W., Filippenko, A. V., Foley, R. J., \& Chornock, R. 2005b, ApJ, 632, 450

Leonard, D. C., et al. 2006, Nature, 440, 505

Li, W. et al. 2007, CBET, 1041, 1

Maeda, K., et al. 2002, ApJ, 565, 405

Maeda, K., Mazzali, P. A., \& Nomoto, K., 2006, ApJ, 645, 1331

Maeda, K., et al. 2007, ApJ, 666, 1069

Maeda, K., et al. 2008, Science, 29, 1220

Massey, P., Strobel, K., Barnes, J. V., \& Anderson, E. 1988, ApJ, 328,315

Maund, J. R., Wheeler, J. C., Patat, F., Baade, D., Wang, L., \& Höflich, P. MNRAS, 381, 201

Maund, J. R., Wheeler, J. C., Patat, F., Baade, D., Wang, L., \& Höflich, P. 2007, A\&A, 475, L1

Mazzali, P. A., et al. 2002, ApJ, 572, L61

Mazzali, P. A., et al. 2005, Science, 308, 1284

Modjaz, M., Kirshner, R.P., \& Challis, P. 2008, submitted to ApJ Letters (arXiv:0801.0221)

Nomoto, K., Yamaoka, H., Pols, O. R., van den Heuvel, E. P. J., Iwamoto, K., Kumagai, S., \& Shigeyama, T. 1994, Nature, 371, 227 
Nomoto, K., Tominaga, N., Tanaka, M., Maeda, K., Suzuki, T., Deng, J. S., \& Mazzali, P. A. 2007, SWIFT and GRBs: Unveiling the Relativistic Universe, Il Nuovo Cimento, 121, 1207-1222 (astroph/0702472)

Oke, J. B. 1990, AJ, 99, 1621

Patat, F., et al. 2001, ApJ, 555, 900

Patat, F. \& Romaniello, M., 2006, PASP, 118, 146

Pilyugin, L. S., Vílchez, J. M., \& Contini, T. 2004, A\&A, 425, 849

Scarrott, S. M., Ward-Thompson, D., \& Warren-Smith, R. F. 1987, MNRAS, 224, 299

Scarrott, S. M., Draper, P. W., Stockdale, D. P., \& Wolstencroft, R. D., 1993, MNRAS, 264, L7

Schmidt, B., Kirshner, R. P., \& Eastman, R. G. 1992, ApJ, 395, 366

Serkowski, K., Mathewson, D. L., \& Ford, V. L. 1975, ApJ, 196, 261

Shapiro, P. R., \& Sutherland, P. G. 1982, ApJ, 263, 902

Silbermann, N. A., et al. 1996, ApJ, 470, 1

Tanaka, M., Maeda, K., Mazzali, P. A., \& Nomoto, K. 2007, ApJ, 668, L19

Terry, J. N., Paturel, G., \& Ekholm, T. 2002, A\&A, 393, 57
Tinbergen, J. 1996, Astronomical Polarimetry (New York: Cambridge Univ. Press)

Tominaga, N., et al. 2005, ApJ, 633, L97

Trammell, S. R., Hines, D. C., \& Wheeler, J. C. 1993, ApJ, 414, L21

Valenti, S., et al. 2008, ApJ, 673, L155

Wang, L., et al. 2001, ApJ, 550, 1030

Wang, L., et al. 2002, ApJ, 579, 671

Wang, L. et al. 2003a, ApJ, 591, 1110

Wang, L., Baade, D., Höflich, P., \& Whheler, J. C 2003b, ApJ, 592, 457

Wang, L. 2004, Cosmic explosions in three dimensions : asymmetries in supernovae and gamma-ray bursts, ed. by P. Hoflich, et al., Cambridge University Press, 17

Wheeler, J. C., Harkness, R. P., Barker, E. S., Cochran, A. L., \& Eills, D. 1987, ApJ, 313, L69

Whittet, D. C. B., Martin, P. G., Hough, J. H., Rouse, M. F., Bailey, J. A., \& Axon, D. J. 1992, ApJ, 386, 562 